\title{
CLA-DE: A Hybrid Model Based On Cellular Learning Automata for Numerical Optimization
}

\author{
R. Vafashoar, M. R. Meybodi, A. H. Momeni \\ Soft Computing Laboratory, Computer Engineering and Information Technology \\ Department, Amirkabir University of Technology, Tehran, Iran.
}

Tel: +98-21-64545120; Fax: +98-21-66495521; e-mail: (vafashoar, mmeybodi,

a_h_momeni)@aut.ac.ir

\begin{abstract}
This paper presents a hybrid model named: CLA-DE for global numerical optimization. This model is based on cellular learning automata (CLA) and differential evolution algorithm. The main idea is to learn the most promising regions of the search space using cellular learning automata. Learning automata in the CLA iteratively partition the search dimensions of the problem and learn the most admissible partitions. In order to facilitate corporation among the CLA cells and improve their impact on each other, differential evolution algorithm is incorporated, by which communication and information exchange among neighboring cells are speeded up. The proposed model is compared with some evolutionary algorithms to demonstrate its effectiveness.

Experiments are conducted on a group of benchmark functions which are commonly used in the literature. The results show that the proposed algorithm can achieve near optimal solutions in all cases, which are highly competitive with the ones from compared algorithms.
\end{abstract}

Keyword

Cellular learning automata; learning automata; optimization; differential evolution algorithm;

\section{Introduction}

Global optimization problems are one of the major challenging tasks in almost every field of science. Analytical methods are not applicable in most cases; therefore, various numerical methods such as evolutionary algorithms [1] and learning automata based methods [2, 3] have been proposed to solve these problems by estimating the global optima. Most of these methods suffer from convergence to local optima and slow convergence rate.

Learning automata (LA) follows the general schemes of reinforcement learning (RL) algorithms. RL algorithms are used to enable agents learn from the 
experiences gained by their interaction with an environment. For more theoretical information and applications of reinforcement learning see [4-6]. Learning automata are used to model learning systems. They operate in a random unknown environment by selecting and applying actions via a stochastic process. They can learn the optimal action by iteratively acting and receiving stochastic reinforcement signals from the environment. These stochastic signals or responses from the environment exhibit the favorability of the selected actions, and according to them a learning automaton modifies its action selecting mechanism in favor of the most promising actions [7-10]. Learning automata have been applied to a vast variety of science and engineering applications [10, 11], including various optimization problems $[12,13]$. A learning automata approach is used for a special optimization problem, which can be formulated as a special knapsack problem [13]. In this work a new on-line Learning Automata System is presented for solving the problem. Sastry et al. used a stochastic optimization method based on continuous-action-set automata for learning a hyperplane classifier which is robust to noise [12]. In spite of its effectiveness in various domains, learning automata have been criticized for having a slow convergence rate.

Meybodi et al. [14] introduced a new model obtained from the combination of learning automata and cellular automata (CA), which they called cellular learning automata (CLA). It is a cellular automaton that one or more learning automata reside in each of its cells. Therefore, the cells of CLA have learning abilities and also can interact with each other in a cellular manner. Because of its learning capabilities and cooperation among its elements, this model is considered superior to both CA and LA. Because of local interactions, cellular models can be implemented on distributed and parallel systems. Up to now, CLA has been applied to many problems. Esnaashari and Meybodi proposed a fully distributed clustering algorithm, based on cellular learning automata, for sensor networks [15]. They also used cellular learning automata for scheduling the active times of the nodes in Wireless Sensor Networks [16]. A Dynamic Web Recommender System Based on Cellular Learning Automata is introduced in [17]. More information about the theory and some applications of CLA can be found in [14, $18,19]$. 
These two paradigms (LA and CLA) have also been used to tackle global numerical optimization problems. Zeng and Liu [3] have used learning automata for continuously dividing and sampling the search space. Their algorithm gradually concentrates on favorable regions and leaves out others. Beigy and Meybodi proposed a new learning algorithm for continuous action learning automata [20] and proved its convergence in stationary environments. They also showed the applicability of the introduced algorithm in solving noise corrupted optimization problems. An evolutionary inspired learning method called genetic learning automata was introduced in 2002 [2]. In this hybrid method, genetic algorithm and learning automata are combined to compensate the drawbacks of both methods. Genetic learning automata algorithm evolves a population of probability strings for reaching the global optima. At each generation, each probability string gives rise to one bit string child. These children are evaluated using a fitness function and based on this evaluation, probability strings are adjusted. After that, these probability strings are further maturated using standard evolutionary operators. A somehow closer approach termed CLA-EC [21] is developed on the basis of cellular learning automata and evolutionary computing paradigm. Learning automata technique is also used for improving the performance of PSO algorithm by adaptively tuning its parameters [22].

Differential evolution algorithm (DE) is one of the recent evolutionary methods that attracted the attention of many researchers. Up to now, after its initial proposal by Storn and Price [23, 24], a great deal of work has been done for its improvement [25-28]. DE is simple, yet very powerful, making it an interesting tool for solving optimization problems in various domains. Some applications of DE can be found in [29]. It is shown [30] that DE has better performance than genetic algorithm (GA) and particle swarm optimization (PSO) over some benchmark functions; still it suffers from premature convergence and stagnation. In the premature convergence, population converges to some local optima and loses its diversity. In stagnation, population ceases approaching global optima whether or not it has been converged to some local ones.

The work presented in this paper is primarily based on cellular learning automata, and uses differential evolution algorithm to improve its convergence rate and the accuracy of achieved results. A CLA constitutes the population of the algorithm and is evolved for reaching the optima. Each cell of the CLA is composed of two 
parts that are termed: Candidate Solution and Solution Model. Solution Model represents a model for the desirability of different regions of the problem search space. Candidate Solution is the temporary fittest solution obtained by the cell. The Solution Model is a learning system including a set of learning automata in which each automaton is prescribed to one dimension of the problem search space, and learns the promising regions of that dimension. The Solution Model governs the evolution of its related Candidate Solution. On the other hand, Candidate Solutions also guide the learning process of their Solution Models, through reinforcement signals provided for Solution Models. Unlike DE or GA approaches in which each entity represents a single point of the search space, each Solution Model represents the entire search space, and so trapping on local optima is avoided. The Solution Models interact with each other through a set of local rules. In this way, they can impact on the learning process of each other. DE algorithm is used to transfer information between neighboring Candidate Solutions which increases impact of Solution Models on each other's learning process.

This paper is organized as follows. In Section 2, the principles on which cellular learning automata are based are discussed. Section 3 includes a brief overview of differential evolution algorithm. The CLA-DE algorithm is then described in more details in Section 4. Section 5 contains implementation considerations, simulation results and comparison with other algorithms to highlight the contributions of the proposed approach. Finally, conclusions and future works are discussed in section 6.

\section{Cellular Learning Automata}

Learning automata are adaptive decision making systems that operate in an unknown stochastic environment with the purpose of determining the optimal action for that environment out of a set of actions. The learning process is as follows: at each stage a learning automaton selects one of its actions according to its probability vector and performs it on the environment; the environment evaluates the performance of the selected action to determine its effectiveness, and then provides a response for the learning automaton. The learning automaton uses this response as an input to update its internal action probabilities. By 
repeating this procedure the learning automaton learns to select the optimal action, which leads to desirable responses from the environment $[7,8]$.

The type of the learning automata which is used in this paper belongs to a family of learning automata called variable structure learning automata. This kind of automata can be represented with a six-tuple like $\{\Phi, \alpha, \beta, \mathrm{A}, \mathrm{G}, \mathrm{P}\}$, where $\Phi$ is a set of internal states, $\alpha$ a set of outputs, $\beta$ a set of input actions, A a learning algorithm, G(.): $\Phi \rightarrow \alpha$ a function that maps the current state into the current output, and $\mathrm{P}$ a probability vector that determines the selection probability of a state at each stage. In this definition the current output depends only on the current state. In many cases it is enough to use an identity function as $G$, in this case the terms action and state may be used interchangeably. The core factor in the performance of the learning automata approach is the choice of the learning algorithm. The learning algorithm modifies the action probability distribution vector according to the received environmental response. One of the commonly used updating methods is linear reword-penalty algorithm $\left(L_{R-P}\right)$. Let $a_{i}$ be the action selected at cycle $n$ according to the probability vector at cycle $n, p(n)$; in an environment with two outputs, $\beta \in\{0,1\}$, if the environmental response to this action is favorable $(\beta=0)$, the probability vector is updated according to equation 1 , otherwise (when $\beta=1$ ) it is updated using equation 2 ,

$$
\begin{aligned}
& p_{j}(k+1)= \begin{cases}p_{j}(k)+a\left(1-p_{j}(k)\right) & \text { if } i=j \\
p_{j}(k)(1-a) & \text { if } i \neq j\end{cases} \\
& p_{j}(k+1)= \begin{cases}p_{j}(k)(1-b) & \text { if } i=j \\
\frac{b}{r-1}+p_{j}(k)(1-b) & \text { if } i \neq j\end{cases}
\end{aligned}
$$

where $\mathrm{a}$ and $\mathrm{b}$ are called reward and penalty parameters respectively.

Cellular automata are abstract models for systems consisting of a large number of identical simple components [31]. These components which are called cells are arranged in some regular forms like grid and ring, and have local interactions with each other. CA operates in discrete times called steps; at each time step each cell will have one state out of a limited number of states, states of all cells define the state of the whole CA. The state of a cell at the next time step is determined according to a set of rules and the states of its neighboring cells. Through these rules, CA evolves from an internal state during the time to produce complicated 
patterns of behavior. The ability to produce sophisticated patterns, from an internal state with only local interactions and a set of simple rules, has caused CA to be a popular tool for modeling complicated systems and natural phenomena. There are some neighborhood models defined in literature for CA, the one which is used in this paper is called Von Neumann.

Cellular Learning Automata (CLA) is an abstract model for systems consisting a large number of simple entities with learning capabilities [14]. It is a hybrid model composed of learning automata and cellular automata. It is a cellular automaton in which each cell contains one or more learning automata. The learning automaton (or group of learning automata) residing in each cell determines the cell state. Each cell is evolved during the time based on its experience and the behavior of the cells in its neighborhood. For each cell the neighboring cells comprise the environment of the cell.

Cellular learning automata starts from some initial state (it is the internal state of every cell); at each stage, each automaton selects an action according to its probability vector and performs it. Next, the rule of cellular automaton determines the reinforcement signal (the response) to the learning automata residing in its cells, and based on the received signal each automaton updates its internal probability vector. This procedure continues until the result is obtained.

\section{Differential Evolution Algorithm}

A differential evolution algorithm like other evolutionary algorithms starts with an initial population and evolves it through some generations. At each generation, it uses evolutionary operators: mutation, crossover, and selection to generate a new and probably better population. The population consists of a set of $\mathrm{N}$-dimensional parameter vectors called individuals: Population $=\left\{\mathrm{X}_{1, \mathrm{G}}, \mathrm{X}_{2, \mathrm{G}}, \ldots, \mathrm{X}_{\mathrm{NP}, \mathrm{G}}\right\}$ and $\mathrm{X}_{\mathrm{i}, \mathrm{G}}$ $=\left\{\mathrm{X}_{\mathrm{i}, \mathrm{G}}{ }^{1}, \mathrm{X}_{\mathrm{i}, \mathrm{G}}{ }^{2}, \ldots, \mathrm{X}_{\mathrm{i}, \mathrm{G}}{ }^{\mathrm{N}}\right\}$, where NP is the population size and $\mathrm{G}$ is the generation number. Each individual is an encoding corresponding to a temporary solution of the problem in hand. At first stage, the population is created randomly according to the uniform distribution from the problem search space for better covering of the entire solution space [23, 24].

In each generation $G$, mutation operator creates a mutant vector $V_{i, G}$, for each individual $\mathrm{X}_{\mathrm{i}, \mathrm{G}}$. There are some mutation strategies reported in the literature, three common ones are listed in equations 3, 4 and 5 . 
$D E$ / rand / 1:

$$
V_{i, G}=X_{r 1, G}+F \cdot\left(X_{r 2, G}-X_{r 3, G}\right)
$$

DE / best / 1:

$$
V_{i, G}=X_{b e s t, G}+F \cdot\left(X_{r 1, G}-X_{r 2, G}\right)
$$

$$
\begin{aligned}
& D E / \text { rand }- \text { to - best / 1: } \\
& \qquad V_{i, G}=X_{i, G}+F .\left(X_{\text {best }, G}-X_{i, G}\right)+F .\left(X_{r 1, G}-X_{r 2, G}\right)
\end{aligned}
$$

where $r_{1}, r_{2}$, and $r_{3}$ are three mutually different random integer numbers uniformly taken from the interval $[1, \mathrm{NP}], \mathrm{F}$ is a constant control parameter which lies in the range $[0,2], X_{\text {best, } G}$ is the individual with the maximum fitness value at generation G.

Each mutant vector, $V_{i, G}$ recombines with its respective parent $X_{i, G}$ through crossover operation to produce the final offspring vector $U_{i, G}$. DE algorithms mostly use binomial or exponential crossover of which the binomial one is given in equation 6. In this type of crossover each generated child inherits each of its parameter values from either of the parents.

$$
U_{i, G}^{j}= \begin{cases}V_{i, G}{ }^{j} & \text { if } \operatorname{Rand}(0,1)<C R \text { or } j=\text { irand } \\ X_{i, G}{ }^{j} & \text { otherwise }\end{cases}
$$

$\mathrm{CR}$ is a constant parameter controlling the portion of the offspring inherited from the mutant vector. irand is a random integer generated uniformly from the interval $[1, N]$ for each child to ensure that at least one of its parameter values are taken from the mutant vector. $\operatorname{Rand}(0,1)$ is a uniform random real number generator from the interval $[0,1]$.

Each generated child is evaluated with the fitness function, and based on this evaluation, eiher the child $U_{i, G}$ or its parent $X_{i, G}$ is selected for the next generation as in equation 7.

$$
X_{i, G+1}= \begin{cases}U_{i, G} & \text { if } f\left(U_{i, G}\right)<f\left(X_{i, G}\right) \\ X_{i, G} & \text { otherwise }\end{cases}
$$




\section{The proposed model}

This Section describes in detail the proposed model to solve the global optimization problem defined as:

$$
\text { Minimize: } y=f(x) \text {, }
$$

Subject to: $\mathrm{x}_{\mathrm{d}} \in\left[\mathrm{s}_{\mathrm{d}}, \mathrm{e}_{\mathrm{d}}\right],(8)$

where $\mathrm{s}=\left[\mathrm{s}_{1}, \mathrm{~s}_{2}, \ldots, \mathrm{s}_{\mathrm{d}}, \ldots, \mathrm{s}_{\mathrm{N}}\right]$ and $\mathrm{e}=\left[\mathrm{e}_{1}, \mathrm{e}_{2}, \ldots, \mathrm{e}_{\mathrm{d}}, \ldots, \mathrm{e}_{\mathrm{N}}\right]$ define the feasible solution space, $x=\left[x_{1}, x_{2}, \ldots, x_{d}, \ldots, x_{N}\right]^{T}$ is a variable vector from this space and $\left[s_{d}, e_{d}\right]$ denotes the domain of the variable $x_{d}$.

Like evolutionary algorithms, in order to reach the final answer, a population of individuals will be evolved through some generations. Each individual is composed of two parts. The first part of an individual is an $\mathrm{N}$ dimensional real vector from the search space, and will be referred to as Candidate Solution. A Candidate Solution represents a probable solution to the problem. The second part which will be called Solution Model is a set of $\mathrm{N}$ learning automata; each one corresponds to one dimension of the Candidate Solution. The Solution Model of an individual governs the evolutionary process of its Candidate Solution. Its objective is to determine the most promising regions of the problem search space by continuously dividing the search space and learning the effectiveness of each division. The population of individuals is spatially distributed on a cellular learning automata. Each cell is occupied by only one individual and each individual is exactly assigned to one cell; therefore, the terms individual and cell can be used interchangeably.

As mentioned earlier, each LA pertains to one dimension of the problem search space and can modify only its associated parameter value in the Candidate Solution. For each individual, $\mathrm{LA}_{d}$ will be used to denote the learning automaton associated to $\mathrm{d}$ dimension. During the evolution process, $\mathrm{LA}_{\mathrm{d}}$ will learn an admissible interval, where the d element of the solution will fall. This interval should be as small as possible in order to achieve a high precision final solution. Figure 1 shows the relationship between the described entities. 


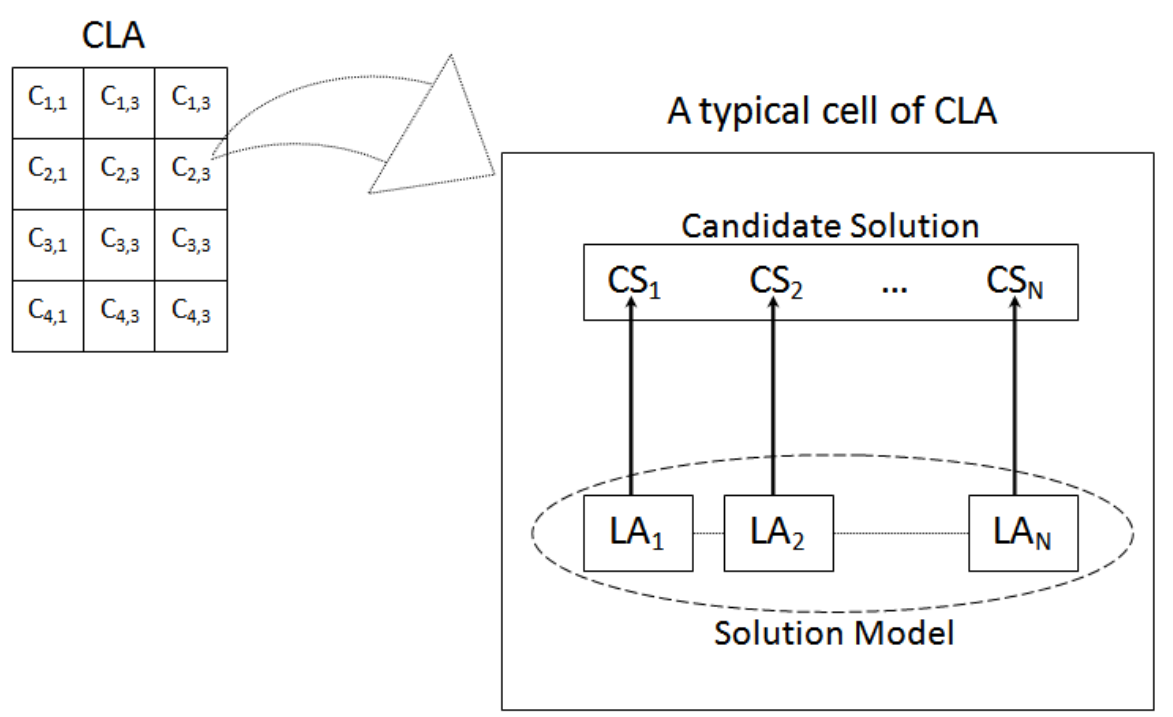

Figure 1 A scheme of CLA-DE model entities

For a typical LA like $\mathrm{LA}_{\mathrm{d}}$, each action corresponds to an interval in its associated domain $\left(\left[\mathrm{s}_{\mathrm{d}}, \mathrm{e}_{\mathrm{d}}\right]\right)$. The actions of $\mathrm{LA}_{\mathrm{d}}$ divide the domain $\mathrm{d}$ into some disjoint intervals in such a way that the union of the intervals equals to the whole domain. These intervals are adapted during the time. At the beginning, the domain is divided into $\mathrm{K}$ intervals with equal length that have no intersections. So at the beginning, each LA has $\mathrm{K}$ actions, and these actions have identical probabilities. During subsequent stages, each LA learns which one of its actions is more appropriate. After that, this action is substituted with two actions whose related intervals are bisections of the former interval and each one's probability is half of the former one's. By continuing this process, the search in the promising regions is refined and the accuracy of the solution is improved. On the other hand, to avoid an unlimited increase in the number of actions, adjacent intervals with small probabilities are merged together, so are their related actions.

CLA cells (individuals) are adapted during a number of iterations. This adaption includes both Solution Model and Candidate Solution parts of an individual. In each cell, some copies of the Candidate Solution are generated. Each learning automaton selects one of its actions and applies it to one of these copies (this process will be explained later). The altered copies generated by a Solution Model are compared to the neighboring ones and based on this comparison, a reinforcement signal is generated for each learning automaton of the Solution Model. Learning automata use these responses to update their action probabilities, and based on these new action probabilities, they may adapt their internal 
structure by action refinement, which will be discussed later. The best generated copy in each cell is used to update the Candidate Solution part.

Each Solution Model modifies its Candidate Solution, and neighboring Candidate Solutions affect the Solution Model by producing reinforcement signals. In this way, information is communicated among the Solution Models. Though this method of communication is rather slow. So in complicated problems, Solution Models tend to learn by themselves and have little impact on the learning behavior of each other. It's desirable to spread good Solution Models into the population for improving convergence and search speed. One way to attain this is to exchange information among the neighboring Candidate Solutions since the Solution Model and the Candidate Solution parts of each cell are correlated. A method based on differential evolutionary algorithm will be used for this purpose, and it will be applied at some generations. The general algorithm is as follows and is described in detail in the subsequent sections.

\section{CLA-DE algorithm:}

1) Initialize the population.

2) While stopping condition is not met do:

3) Synchronously update each cell based on Model Based Evolution.

4) If generation number is a multiple of 5 do:

5) Synchronously update each cell based on DE Based Evolution.

6) End.

7) End.

Figure 2 General pseudo code for CLA-DE

\subsection{Initialization}

At first stage, the population is initiated. The Candidate Solution part of each individual is randomly generated from the search space using the uniform random distribution. Each LA like $\mathrm{LA}_{d}$ will have $\mathrm{K}$ actions with equal probabilities $1 / \mathrm{K}$ at the beginning. These $\mathrm{K}$ actions partition the domain of the dimension $\mathrm{d}$ into $\mathrm{K}$ nonintersecting intervals. For each action $\mathrm{a}_{\mathrm{d}, \mathrm{j}}$, its corresponding interval is defined as in equation 9.

$$
\left[s_{d, j} e_{d, j}\right]=\left[s_{d}+(j-1) \frac{e_{d}-s_{d}}{K} \quad s_{d}+j \frac{e_{d}-s_{d}}{K}\right] \quad, 1 \leq j \leq K
$$




\subsection{Model Based Evolution}

At each iteration of the algorithm, each cell evolves based on its Solution Model and the received signals from its neighboring cells. Consider a cell like $\mathrm{C}$, first its learning automata are partitioned into L disjoint groups (some of these groups may be void). Each group creates one replica of the cell's Candidate Solution. Then, each learning automaton like $\mathrm{LA}_{d}$ in the group selects one of its actions based on its probability vector. Let the selected action of $\mathrm{LA}_{\mathrm{d}}$ be the $\mathrm{j}^{\text {th }}$ action which is associated with an interval like $\left[\mathrm{s}_{\mathrm{d}, \mathrm{j}}, \mathrm{e}_{\mathrm{d}, \mathrm{j}}\right]$; a random number $\mathrm{r}$ is uniformly selected from this interval and the parameter value in the $\mathrm{d}^{\text {th }}$ dimension of the replica is changed to $r$. This way, a group of children is generated for each cell. Figure 3 (a) schematically shows these ideas.

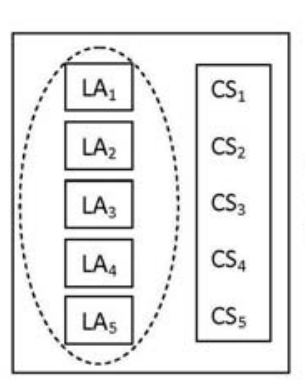

(a)

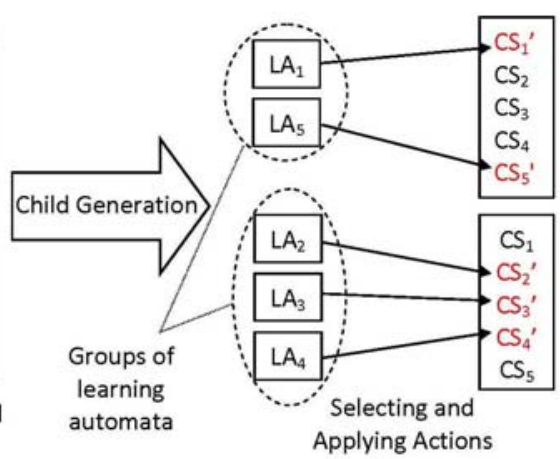

(b)

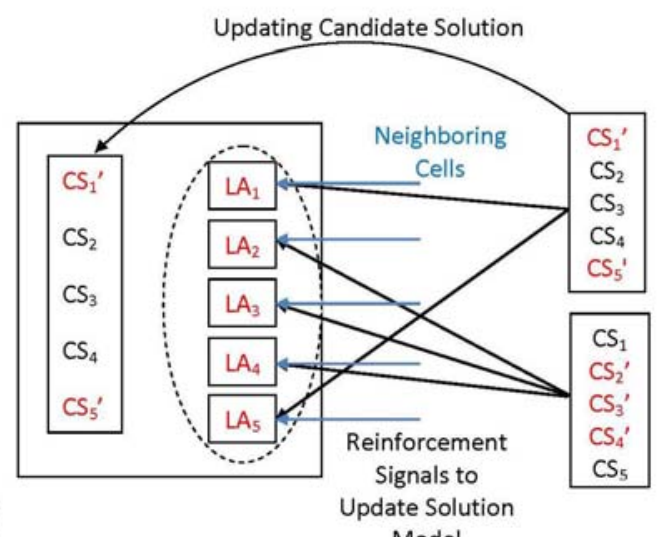

Model

Figure 3 (a) Learning automata of each cell are randomly partitioned into exclusive groups and each group creates one child for the cell. (b) Reinforcement signals from generated children and the neighboring cells are used for updating the Solution Model and the best generated child is used for updating the Candidate Solution. 
The Best generated child in each cell is picked out and is compared with the Candidate Solution of the cell, and the superior one is selected as the Candidate Solution for the next generation. After updating the Candidate Solution part of a cell, its Solution model is also updated. Each selected action of the learning automata is evaluated in the environment and its effectiveness is measured. Consider a typical LA like $\mathrm{LA}_{d}$; its selected action will be rewarded if this action matches more than half of the neighboring Candidate Solutions, or if its corresponding generated child is at least better than the half of the neighboring Candidate Solutions. An action like $a_{d, j}$ that defines an interval like $\left[\mathrm{s}_{\mathrm{d}, \mathrm{j}}, \mathrm{e}_{\mathrm{d}, \mathrm{j}}\right]$ matches a Candidate Solution like $\mathrm{CS}=<\mathrm{CS}_{1}, \mathrm{CS}_{2}, \ldots, \mathrm{CS}_{\mathrm{N}}>$ if $\mathrm{CS}_{\mathrm{d}} \in\left[\mathrm{s}_{\mathrm{d}, \mathrm{j}}, \mathrm{e}_{\mathrm{d}, \mathrm{j}}\right]$. If an action is favorable, then its corresponding LA is updated based on equation 1 and otherwise, it is updated as in equation 2. Figure 3 (b) depicts these steps. Pseudo code for this phase of algorithm is shown in figure 4. Consider that after updating the probability vector of each learning automata, its actions are also refined. The action refinement step is described in the subsequent section.

\section{Model Based Evolution Phase:}

1) For each cell $\mathrm{C}$, with Candidate Solution $\mathrm{CS}=\left\{\mathrm{CS}_{1}, \ldots, \mathrm{CS}_{\mathrm{N}}\right\}$ and Solution Model $\mathrm{M}=\left\{\mathrm{LA}_{1}\right.$, $\left.\ldots, \mathrm{LA}_{\mathrm{N}}\right\}$, where $\mathrm{N}$ is the number of dimensions, do:

2) Randomly partition $M$ into $L$ mutually disjoint groups: $G=\left\{G^{1}, \ldots, G^{L}\right\}$.

3) For Each nonempty Group $G^{i}$ do:

4) Create a copy $\operatorname{CSC}^{\mathrm{i}}=\left\{\operatorname{CSC}_{1}^{\mathrm{i}}, \ldots, \operatorname{CSC}_{\mathrm{N}}^{\mathrm{i}}\right\}$ of $\mathrm{CS}\left(\mathrm{CSC}^{\mathrm{i}}=\mathrm{CS}\right)$, for $\mathrm{G}^{\mathrm{i}}$.

5) For each $\mathrm{LA}_{d} \in \mathrm{G}^{\mathrm{i}}$ associated with the dth dimension of CS do:

6) Select an action from the action set of $\mathrm{LA}_{\mathrm{d}}$ according to its probability. Let this action correspond to an interval like $\left[\mathrm{s}_{\mathrm{d}, \mathrm{j}} \mathrm{e}_{\mathrm{d}, \mathrm{j}}\right]$.

7) Create a uniform random number $r$ from the interval $\left[s_{d, j}, e_{d, j}\right]$ and alter the value of $\operatorname{CSC}_{\mathrm{d}}^{\mathrm{i}}$ to $\mathrm{r}$.

8) End.

9) Evaluate $\mathrm{CSC}^{\mathrm{i}}$ with fitness function.

10) End.

11) End.

12) For each cell $\mathrm{C}$ do:

13) Create a reinforcement signal for each one of its LAs.

14) Update the action probabilities of each LA based on its received reinforcement signal.

16) Refine the actions of each LA in C.

17) End.

Figure 4 pseudo code for Model Based Evolution phase. 


\section{Action Refinement:}

The proposed model slightly differs from other LA based algorithms which have fixed actions. In our proposed model, the actions of learning automata will change during the execution of the algorithm. After updating action probabilities of a typical LA like $\mathrm{LA}_{d}$, if one of its actions like $\mathrm{a}_{\mathrm{d}, \mathrm{j}}$ corresponding to an interval like $\left[\mathrm{s}_{\mathrm{d}, \mathrm{j}}, \mathrm{e}_{\mathrm{d}, \mathrm{j}}\right]$ seems to be more appropriate, then it will be replaced by two actions representing the two halves $\left[\mathrm{s}_{\mathrm{d}, \mathrm{j}},\left(\mathrm{s}_{\mathrm{d}, \mathrm{j}}+\mathrm{e}_{\mathrm{d}, \mathrm{j}}\right) / 2\right]$ and $\left[\left(\mathrm{s}_{\mathrm{d}, \mathrm{j}}+\mathrm{e}_{\mathrm{d}, \mathrm{j}}\right) / 2, \mathrm{e}_{\mathrm{d}, \mathrm{j}}\right]$ of the interval. An action is considered to be appropriate if its probability exceeds a threshold like $\chi$. This will cause finer probing of the promising regions. Dividing an action of a learning automaton has no effect on what has been learned by the LA, since the probability of selecting a subinterval from any desired interval in the pertaining dimension is the same as before.

Replacing one action with two ones causes an increase in the number of actions, and continuing this process would cause the number of actions to increase tremendously. Considering that the sum of the probabilities of all actions of an LA always equals one, therefore an increase in the probability of one action induces a decrease on the probabilities of the other actions of the LA. So there would be some actions with low probabilities that we can merge together making the number of actions almost constant. To attain this, any adjacent actions (whose related intervals are adjacent) with probabilities lower than some threshold like $\varepsilon$ are merged together.

As an example, consider an LA with five actions $\left\{a_{d, 1}, a_{d, 2}, a_{d, 3}, a_{d, 4}, a_{d, 5}\right\}$ with respective probabilities $\{0.05,0.05,0.51,0.35,0.04\}$ and corresponding intervals $\{[1,2),[2,4),[4,4.3),[4.3,5),[5,6)\}$. If $\chi$ and $\varepsilon$ be respectively 0.5 and 0.07 , then after the action refinement step, the LA will be changed as follows. It will have 5 actions $\left\{\mathrm{a}_{\mathrm{d}, 1}, \mathrm{a}_{\mathrm{d}, 2}, \mathrm{a}_{\mathrm{d}, 3}, \mathrm{a}_{\mathrm{d}, 4}, \mathrm{a}_{\mathrm{d}, 5}\right\}$ with corresponding probabilities $\{0.1$, $0.255,0.255,0.35,0.04\}$ and respective intervals $\{[1,4),[4,4.15),[4.15,4.3),[4.3,5)$, $[5,6)\}$. The pseudo code in figure 5 describes the action refinement step. 


\section{Action Refinement Step:}

1) If the probability $\mathrm{p}_{\mathrm{d}, \mathrm{j}}$ of an action $\mathrm{a}_{\mathrm{d}, \mathrm{j}}$ corresponding to an interval $\left[\mathrm{s}_{\mathrm{d}, \mathrm{j}}, \mathrm{e}_{\mathrm{d}, \mathrm{j}}\right]$ is greater than $\chi$, split the action into two actions $\mathrm{a}_{\mathrm{d}, \mathrm{j}}{ }^{1}$ and $\mathrm{a}_{\mathrm{d}, \mathrm{j}}{ }^{2}$ with the corresponding intervals $\left[\mathrm{s}_{\mathrm{d}, \mathrm{j}},\left(\mathrm{s}_{\mathrm{d}, \mathrm{j}}+\mathrm{e}_{\mathrm{d}, \mathrm{j}}\right) / 2\right]$ and $\left[\left(\mathrm{s}_{\mathrm{d}, \mathrm{j}}+\mathrm{e}_{\mathrm{d}, \mathrm{j}}\right) / 2, \mathrm{e}_{\mathrm{d}, \mathrm{j}}\right]$ and equal probabilities $\mathrm{p}_{\mathrm{d}, \mathrm{j}} / 2$.

2) If the probabilities of two adjacent actions $a_{d, j}$ and $a_{d, j+1}$ with corresponding regions $\left[s_{d, j} e_{i, j}\right]$ and $\left[\mathrm{e}_{\mathrm{d}, \mathrm{j}} \mathrm{e}_{\mathrm{i}, \mathrm{j}+1}\right]$ are both less than $\varepsilon$, merge them into one action $\mathrm{a}_{\mathrm{d}, \mathrm{j}}$ ' with corresponding interval: $\left[\mathrm{s}_{\mathrm{d}, \mathrm{j}}\right.$ $\left.\mathrm{e}_{\mathrm{d}, \mathrm{j}+1}\right]$, and probability $\mathrm{p}_{\mathrm{d}, \mathrm{j}}+\mathrm{p}_{\mathrm{d}, \mathrm{j}+1}$.

Figure 5 pseudo code for action refinement step

\subsection{DE Based Evolution}

In this phase, each Candidate Solution is evolved based on equations 3, 4 and 6; though, the process slightly differs from that of the standard DE algorithm. In our model, instead of producing just one new child for each Candidate Solution, several children are generated, and then the best one is selected for updating the Candidate Solution. In order to do this, four Candidate Solutions are selected without replacement from the neighborhood of each cell. The best one is considered as $\mathrm{P}_{\text {best }}$ and others as $\mathrm{P}_{1}, \mathrm{P}_{2}$ and $\mathrm{P}_{3}$. Using different combinations of $\mathrm{P}_{1}$, $\mathrm{P}_{2}$ and $\mathrm{P}_{3}$ a set of mutant vectors are generated based on equation 3. Based on equation 4, another set of mutant vectors are also generated with different combinations of $\mathrm{P}_{1}, \mathrm{P}_{2}$ and $\mathrm{P}_{3}$ in the deferential term and $\mathrm{P}_{\text {best }}$ in the base term. The set of generated mutant vectors are then recombined with the Candidate Solution of the cell based on equation 6 to generate the children set. A pseudo code describing this phase is shown in figure 6. 


\section{DE Based Evolution Phase:}

1) For each cell $\mathrm{C}$ with Candidate Solution $\mathrm{CS}=\left\{\mathrm{CS}_{1}, \ldots, \mathrm{CS}_{\mathrm{N}}\right\}$ do (where $\mathrm{N}$ is the number of dimensions) :

2) Let $\mathrm{P}_{\text {best }}, \mathrm{P}_{1}, \mathrm{P}_{2}$ and $\mathrm{P}_{3}$ be four mutually different Candidate Solutions in the neighborhood of $\mathrm{C}$, where $\mathrm{P}_{\text {best }}$ is the best Candidate Solution neighboring $\mathrm{C}$.

3) Create 12 children $U=\left\{U^{1}, U^{2}, \ldots, U^{12}\right\}$ for $C S, U^{i}=\left\{U_{1}^{i}, U^{i}, \ldots, U^{i}\right\}$ :

$$
\begin{aligned}
& \mathrm{V}_{1: 6}=\left\{\mathrm{P}_{\mathrm{i}}+\mathrm{F} \times\left(\mathrm{P}_{\mathrm{j}}-\mathrm{P}_{\mathrm{k}}\right) \mid \mathrm{i}, \mathrm{j}, \mathrm{k}=1,2,3\right\}, \\
& \mathrm{V}_{6: 12}=\left\{\mathrm{P}_{\text {best }}+\mathrm{F} \times\left(\mathrm{P}_{\mathrm{i}}-\mathrm{P}_{\mathrm{j}}\right) \mid \mathrm{i}, \mathrm{j}=1,2,3\right\},
\end{aligned}
$$

Recombine each $\mathrm{V}_{1: 12}$ with $\mathrm{CS}$ based on eq. 6 to form $\mathrm{H}$ :

$$
U_{i, j}= \begin{cases}V_{i, j} & \text { if } \operatorname{rand}(0,1)<C R \text { or } j=\text { irand } \\ X_{i, j} & \text { otherwise }\end{cases}
$$

4) Select the best generated child $U_{\text {best }}$ according to fitness function.

5) If $U_{\text {best }}$ is better than CS replace CS with $U_{\text {best }}$.

Figure 6 pseudo code for DE Based Evolution

\section{Implementation and numerical results}

In order to evaluate the performance of the proposed model, it is applied to 11 benchmark functions that are shown in table 1 with some of their key properties. These functions include unimodal and multimodal functions with correlated and uncorrelated variables and are vastly used in the literature for examining the reliability and effectiveness of algorithms [32, 33]. In unimodal functions, the convergence rate is the most interesting factor of the study, and multimodal ones are incorporated to study the capability of algorithms in escaping from local

\begin{tabular}{|c|c|c|c|c|}
\hline Equation & $\begin{array}{l}\text { Search } \\
\text { domain }\end{array}$ & $\begin{array}{l}\text { Problem } \\
\text { dimension }\end{array}$ & $\begin{array}{l}\text { Known } \\
\text { global } \\
\text { value }\end{array}$ & $\begin{array}{c}\text { Max } \\
\text { precision }\end{array}$ \\
\hline$f_{1}=\sum_{i=1}^{N}\left(-x_{i} \sin \left(\sqrt{\left|x_{i}\right|}\right)\right)$ & {$[-500,500]^{\mathrm{N}}$} & 30 & -12569.49 & - \\
\hline$f_{2}=\sum_{i=1}^{N}\left(x_{i}^{2}-10 \cos \left(2 \pi x_{i}\right)+10\right)$ & {$[-5.12,5.12]^{\mathrm{N}}$} & 30 & 0 & $1 \mathrm{e}-14$ \\
\hline
\end{tabular}
optima and achieving global optima.

Table 1 benchmark functions and their properties 


$$
\begin{aligned}
& f_{3}=-20 \exp \left(-0.2 \sqrt{\frac{1}{N} \sum_{i=1}^{N} x_{i}^{2}}\right) \\
& -\exp \left(\frac{1}{N} \sum_{i=1}^{N} \cos \left(2 \pi x_{i}\right)\right)+20+\exp (1) \\
& f_{4}=\frac{1}{4000} \sum_{i=1}^{N} x_{i}^{2}-\prod_{i=1}^{N} \cos \left(\frac{x_{i}}{\sqrt{i}}\right)+1 \\
& f_{5}=\frac{\pi}{N}\left\{\begin{array}{l}
\sum_{i=1}^{N-1}\left(y_{i}-1\right)^{2}\left[1+10 \sin ^{2}\left(\pi y_{i+1}\right)\right] \\
+10 \sin ^{2}\left(\pi y_{1}\right)+\left(y_{N}-1\right)^{2}
\end{array}\right\} \\
& +\sum_{i=1}^{N} u\left(x_{i}, 10,100,4\right) \\
& \text { where: } y_{i}=1+1 / 4\left(x_{i}+1\right) \\
& \text { and }: u\left(x_{i}, a, k, m\right)=\left\{\begin{array}{l}
k\left(x_{i}-a\right)^{m}, x_{i}>a \\
0,-a \leq x_{i} \leq a \\
k\left(-x_{i}-a\right)^{m}-a<x_{i}
\end{array}\right. \\
& f_{6}=\sum_{i=1}^{N} u\left(x_{i}, 5,100,4\right)+ \\
& \frac{1}{10}\left\{\begin{array}{l}
\sum_{i=1}^{N-1}\left(x_{i}-1\right)^{2}\left[1+\sin ^{2}\left(3 \pi x_{i+1}\right)\right] \\
+\sin ^{2}\left(3 \pi x_{1}\right)+\left(x_{N}-1\right)^{2}\left[1+\sin ^{2}\left(2 \pi x_{N}\right)\right]
\end{array}\right\} \\
& {[-50,50]^{\mathrm{N}}} \\
& 30 \\
& 0 \\
& \text { 1e-30 } \\
& \text { with } u \text { defined } a \mathrm{~s} \text { in } f_{11} \\
& f_{7}=-\sum_{i=1}^{N} \sin \left(x_{i}\right) \sin ^{20}\left(\frac{i \times x_{i}^{2}}{\pi}\right) \\
& f_{8}=\frac{1}{N} \sum_{i=1}^{N}\left(x_{i}^{4}-16 x_{i}^{2}+5 x_{i}\right) \\
& f_{9}=\sum_{j=1}^{N-1}\left[100\left(x_{j}-x_{j+1}\right)^{2}+\left(x_{j}-1\right)^{2}\right] \\
& f_{10}=\sum_{i=1}^{N} x_{i}^{2} \\
& f_{11}=\sum_{i=1}^{N}\left|x_{i}\right|+\prod_{i=1}^{N}\left|x_{i}\right| \\
& {[-0, \pi]^{\mathrm{N}} \quad 100 \quad-99.2784} \\
& {[-5,5]^{\mathrm{N}} \quad 100 \quad-78.33236} \\
& 30 \\
& 0
\end{aligned}
$$




\subsection{Algorithms for comparison and simulation settings}

\section{Algorithms for comparison and their settings:}

To evaluate the effectiveness of the proposed model, it is compared to four other evolutionary algorithms. These algorithms include: DE/rand/bin/ $/{ }^{1}$, DERL [25], ODE [27] and PSO with inertia weight (PSO-W) [34].

The population size NP is equal to 100 for DE/rand/bin/1, DERL, and ODE. This population size is used and recommended in many works for 30 dimensional problems $[26,27]$. NP $=10 \times \mathrm{N}$, is used in the original paper for DERL [25], and is recommended in some other works. But almost on all of the used benchmark functions DE algorithms, including DERL, have much better performance with $\mathrm{NP}=100$, so the population size of 100 is adopted for all DE algorithms. There are 2 100-dimensional functions in table 1, larger population sizes are also tested for these functions, but better results achieved when we used NP $=100$. For ODE and $\mathrm{DE} / \mathrm{rand} / \mathrm{bin} / 1, \mathrm{~F}$ and $\mathrm{CR}$ are set to the values 0.5 and 0.9 respectively which are used in many works $[26,27,30,36]$. RLDE uses $C R=0.5$ along with a random $\mathrm{F}$ value for each generated mutant vector. The values for parameter $\mathrm{F}$ are taken uniformly from one of the intervals $[-1,-0.4]$ or $[0.4,1][25]$.

The PSO algorithm is implemented as described in [34]. In this implementation, an inertia weight (w) is considered for velocity which is linearly decreased from 0.9 to 0.4 in each generation. The parameters $\mathrm{C} 1$ and $\mathrm{C} 2$ are kept 2 as used in [34].

\section{Parameter settings for CLA-DE:}

Many experiments are conducted to study the impact of different parameter settings on the performance of the proposed model, and accordingly, the following settings are adopted. $3 \times 4$ CLA with $\mathrm{L}_{\mathrm{R}-\varepsilon \mathrm{P}}$ learning algorithm for $\mathrm{LAs}$ are used; population size is kept small in order to let the CLA learn for more generations and to demonstrate its learning capability. $\mathrm{K}$, number of initial actions, is set to be 5 . At each iteration, $M$ is portioned into $N / 2$ groups $(L=N / 2)$, so in the average case each group contains two Learning automata. Large values for L would cause LA's to learn individually, which may cause failure in achieving a desirable result for correlated problems, though small values for $\mathrm{L}$ are

\footnotetext{
${ }^{1}$ Source code for DE is publicly available at: http://www.icsi.berkeley.edu/ storn/code.html
} 
also inappropriate because with large groups of cooperating LA's it is difficult to evaluate the performance of each LA. Probability thresholds for dividing and merging actions, $\chi$ and $\varepsilon$, are reasonably set to the values: two times the initial action probability and some less than half of the initial action probability e.g.: 0.4 and 0.07 . DE based evolution part parameters, i.e. F and CR, are set to the values: 0.8 and 0.9 as recommended in [35]. DE based evolution is repeated every 5 generations.

\subsection{Experiment 1: comparison of finding the global optimum}

In this section the effectiveness of the tested algorithms in reaching the global optima is studied. The success rate of each algorithm on each benchmark is determined. A run of an algorithm on a benchmark is considered to be successful, if the difference of the reached solution and the global optimum is less than a threshold $\Delta$. A run of an algorithm is terminated after the success occurrence or after 300000 function evaluations. The average number of function evaluations (FE) for the successful runs is also determined for each algorithm. Success rate and average number of function evaluations are used as the criteria for comparison. For all benchmark functions, except $f_{7}$ and $f_{9}$, the accuracy of the achieved results or $\Delta$ is considered 1e-5. None of the compared algorithms have reached this accuracy on the functions $\mathrm{f}_{7}$ and $\mathrm{f}_{9}$ (table 4 ), so 60 and 0.1 are used as $\Delta$ for $f_{7}$ and $f_{9}$ respectively. The success rate along with the average number of function evaluations for each algorithm on the given benchmarks is shown in table 2. Based on the results of table 2 , table 3 shows the success rate and average number of function evaluation ranks of each method. All results reported in this and next sections are obtained based on 40 independent trials.

The first point which is obvious form the achieved results is the robustness of the proposed approach. This issue is apparent from the success rates of CLA-DE which, except in one case, are the highest of all compared ones. Considering problems $\mathrm{f}_{2}, \mathrm{f}_{7}$ and $\mathrm{f}_{8}$ CLA-DE is the only algorithm that effectively solved these problems. Although CLA-DE didn't achieve the best results in terms of average number of function evaluations in all cases, but it has an acceptable FE in all cases. CLA-DE has a better performance than DE and DERL in terms of approaching speed to the global optima in almost all of the cases. Although it has a weaker performance than ODE on some benchmarks, according to this criterion, 
but CLA-DE has a high success rate in comparison to ODE. Considering table 3, the success rate rank of ODE is a bit lower than DE and DERL, which is the result of its fast convergence rate.

Table 2 Mean success rate and Mean number of function evaluations to reach the specified accuracy for successful runs. Success rates are shown inside the parentheses

\begin{tabular}{|c|c|c|c|c|}
\hline & CLA-DE & $\overline{\mathrm{DE}}$ & DERL & ODE \\
\hline$f_{1}$ & $71600(1)$ & $-(0)$ & $184242.50(1)$ & $-(0)$ \\
\hline $\mathrm{f}_{2}$ & $102256.6(1)$ & - $(0)$ & $-(0)$ & $115147(0.05)$ \\
\hline $\mathrm{f}_{3}$ & $116616.6(\mathbf{1})$ & $124688.8(\mathbf{1})$ & $143642.5(\mathbf{1})$ & $69087.5(1)$ \\
\hline $\mathrm{f}_{4}$ & 90455.5 (1) & $93902.3(0.95)$ & $111670(\mathbf{1})$ & $53958.9(0.97)$ \\
\hline$f_{5}$ & $50496.6(\mathbf{1})$ & 80140.4 (1) & 104097.5 (1) & $41220(1)$ \\
\hline $\mathrm{f}_{6}$ & $60430(1)$ & 88048.3 (1) & $109680(\mathbf{1})$ & $40052.5(1)$ \\
\hline $\mathrm{f}_{7}$ & 3570 (1) & $-(0)$ & $-(0)$ & $233263.6(0.55)$ \\
\hline $\mathrm{f}_{8}$ & $179595(1)$ & $267199(0.1)$ & $298807(0.02)$ & $-(0)$ \\
\hline $\mathrm{f}_{9}$ & $246218(0.64)$ & $238117(0.9)$ & $266219(0.05)$ & $247251(0.05)$ \\
\hline$f_{10}$ & 80595 (1) & 89956.4 (1) & 101243 (1) & $46147.5(1)$ \\
\hline$f_{11}$ & $113265(\mathbf{1})$ & $135922.3(\mathbf{1})$ & $135055(\mathbf{1})$ & 106115 \\
\hline
\end{tabular}

Table 3 Success rate and Mean number of function evaluation ranks of the compared methods. Success rate ranks are shown inside the parentheses

\begin{tabular}{ccccccccccccc}
\hline & $\mathrm{f}_{1}$ & $\mathrm{f}_{2}$ & $\mathrm{f}_{3}$ & $\mathrm{f}_{4}$ & $\mathrm{f}_{5}$ & $\mathrm{f}_{6}$ & $\mathrm{f}_{7}$ & $\mathrm{f}_{8}$ & $\mathrm{f}_{9}$ & $\mathrm{f}_{10}$ & $\mathrm{f}_{11}$ & $\begin{array}{c}\text { Average } \\
\text { rank }\end{array}$ \\
\hline CLA-DE & $1(1)$ & $1(1)$ & $2(1)$ & $2(1)$ & $2(1)$ & $2(1)$ & $1(1)$ & $1(1)$ & $2(2)$ & $2(1)$ & $2(1)$ & $1.63(1.09)$ \\
DE & $3(2)$ & $3(3)$ & $3(1)$ & $3(3)$ & $3(1)$ & $3(1)$ & $3(3)$ & $2(2)$ & $1(1)$ & $3(1)$ & $3(1)$ & $2.72(1.72)$ \\
DERL & $2(1)$ & $3(3)$ & $4(1)$ & $4(1)$ & $4(1)$ & $4(1)$ & $3(3)$ & $3(3)$ & $4(3)$ & $4(1)$ & $4(1)$ & $3.54(1.72)$ \\
ODE & $3(2)$ & $2(2)$ & $1(1)$ & $1(2)$ & $1(1)$ & $1(1)$ & $2(2)$ & $4(4)$ & $3(3)$ & $1(1)$ & $1(1)$ & $1.81(1.81)$ \\
\hline
\end{tabular}

\subsection{Experiment 2: comparison of mean best fitness}

This experiment is conducted for investigating the mean best fitness that the compared algorithms could achieve after a fixed number of fitness function evaluations. For a fair comparison, all runs of an algorithm are terminated after 300000 function evaluations, or when the error of achieved result falls below some specified precision (table 1) for some particular functions. These threshold 
values are used because variable truncations in implementations limit the precision of results in the cases of these functions.

Evolution curves (the mean best versus the fitness evaluations) for all of the algorithms described in section 5.1 are depicted in figures 7 and 8; in addition, table 4 shows the average final result along with its standard deviation for the different algorithms.

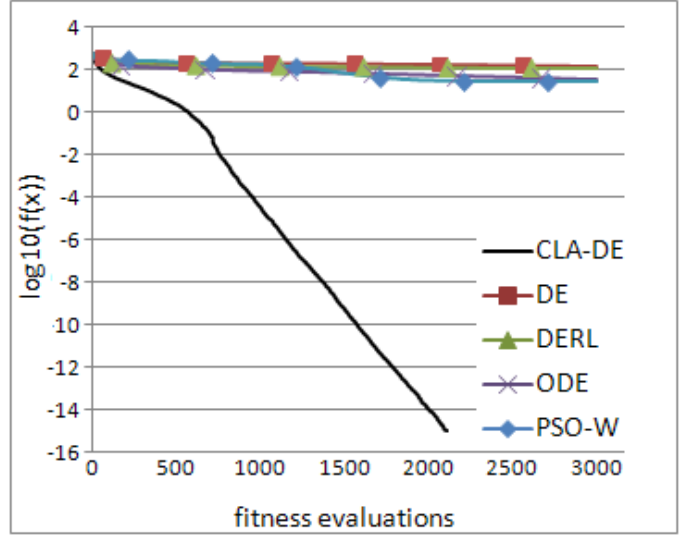

(a)

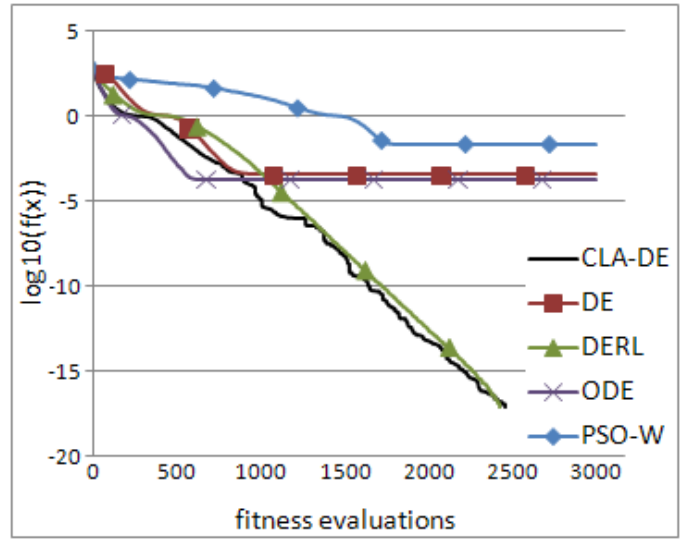

(c)

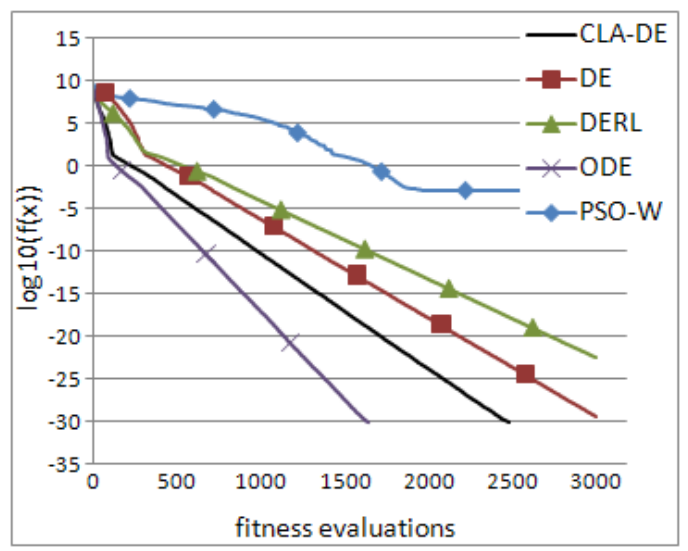

(e)

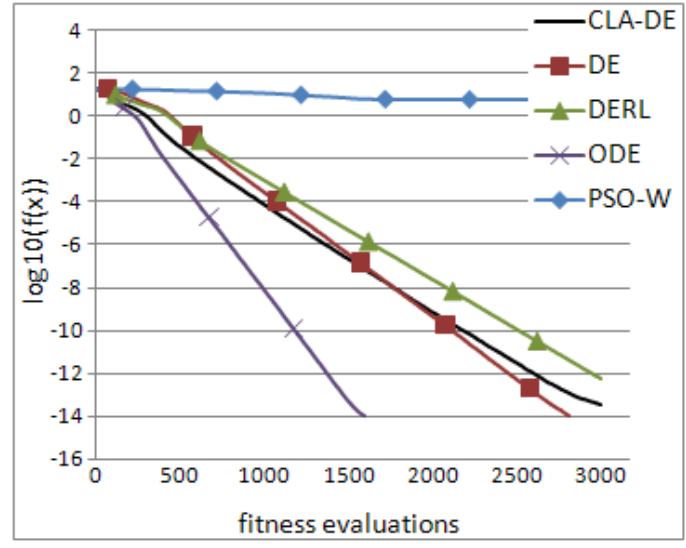

(b)

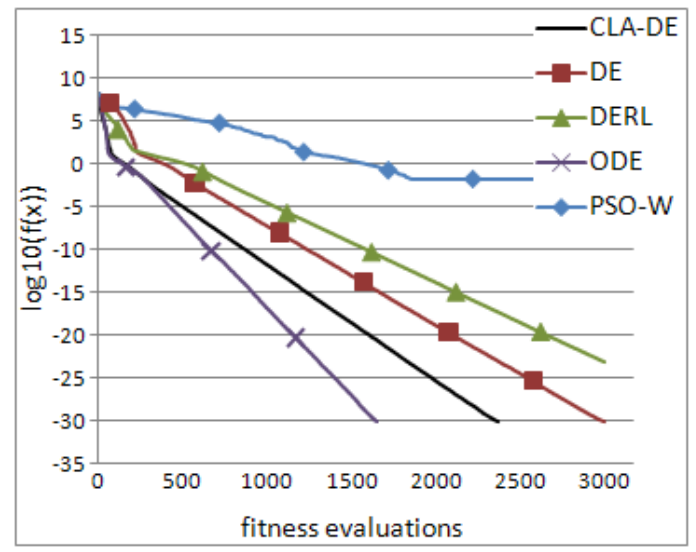

(d)

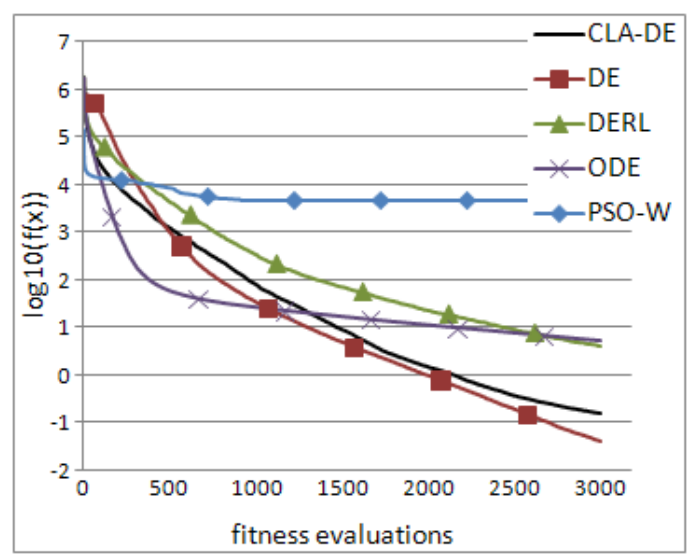

(f) 


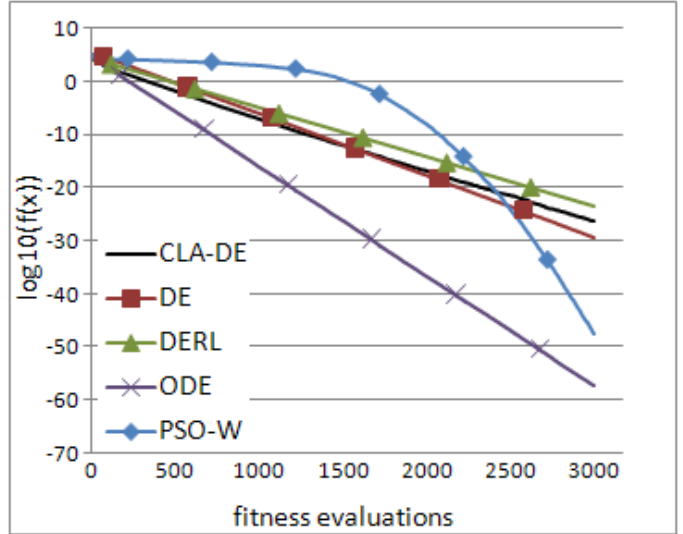

(g)

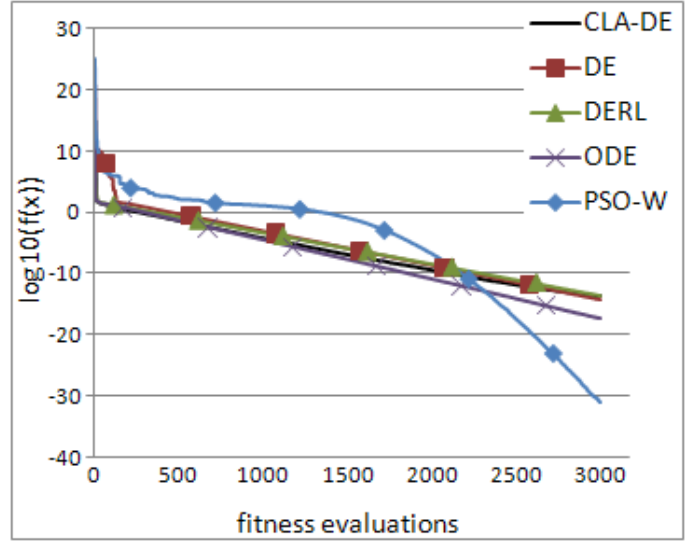

(h)

Figure 7 Fitness curves for functions (a) $f_{2}$, (b) $f_{3}$, (c) $f_{4}$, (d) $f_{5}$, (e) $f_{6}$, (f) $f_{9},(g) f_{10}$ and (h) $f_{11}$. The horizontal axis is the number of fitness evaluations and the vertical axis is the logarithm in base 10 of mean best function value over all trials.

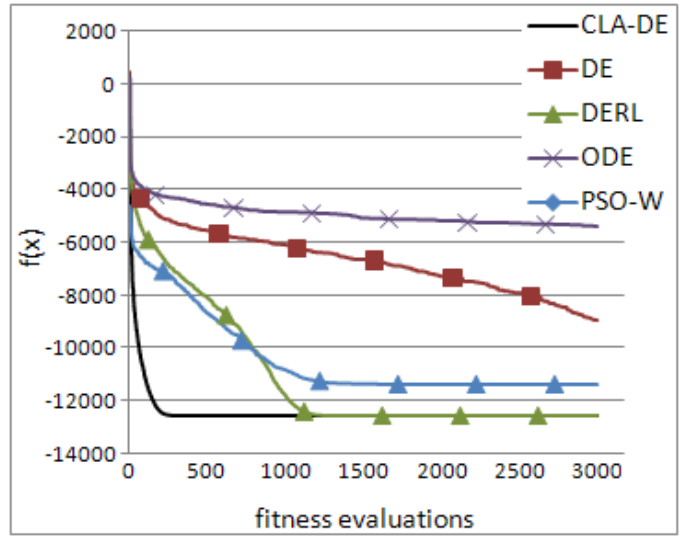

(a)

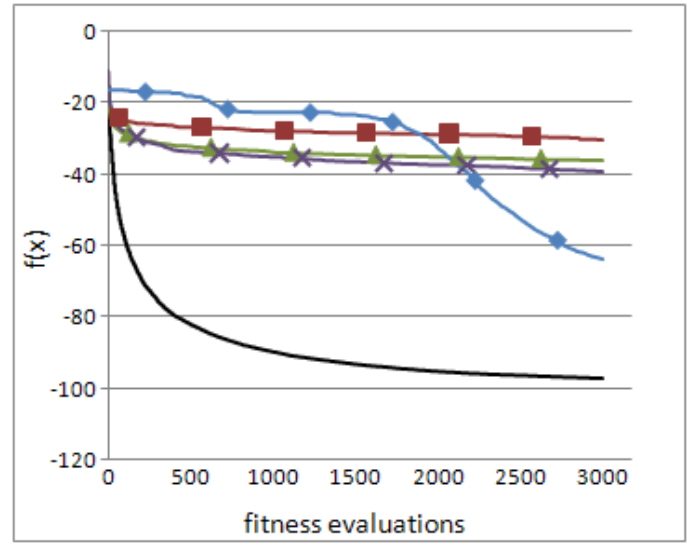

(b)

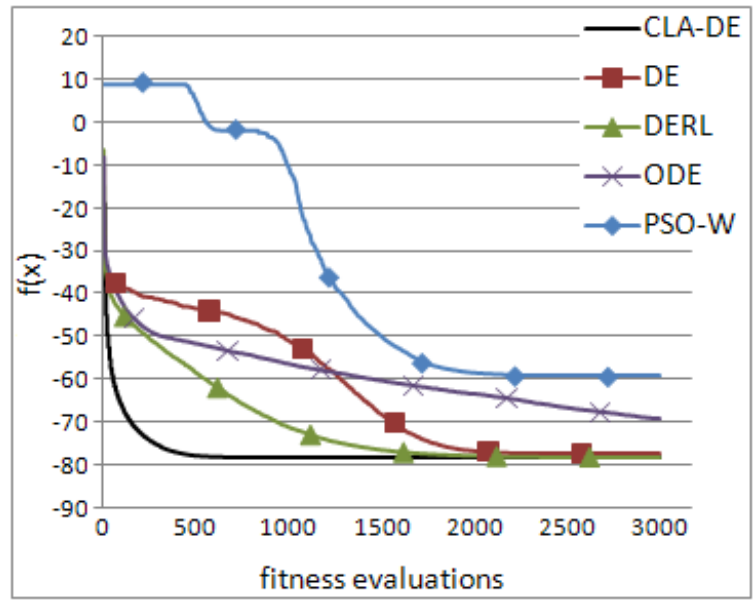

(c)

Figure 8 Fitness curves for functions (a) $\mathrm{fl}$, (b) $\mathrm{f} 7$ and (c) $\mathrm{f} 8$. The horizontal axis is the number of fitness evaluations and the vertical axis is the mean best function value over all trials. 
Table 4 average final result (along with its standard deviation)

\begin{tabular}{cccccc}
\hline & CLA-DE & PSO-W & DE & DERL & ODE \\
\hline $\mathrm{f}_{1}$ & $\mathbf{- 1 2 5 6 9 . 4 9}$ & -11367.10 & -8940.19 & $\mathbf{- 1 2 5 6 9 . 4 9}$ & -5415.23 \\
& $(3.71 \mathrm{e}-12)$ & $(8.43 \mathrm{e}+2)$ & $(1.55 \mathrm{e}+3)$ & $(3.68 \mathrm{e}-12)$ & $(5.35 \mathrm{e}+2)$ \\
$\mathrm{f}_{2}$ & $\mathbf{8 . 7 0 e - 1 5}$ & 26.41 & 133.55 & 117.11 & 32.27 \\
& $(7.15 \mathrm{e}-16)$ & $(8.07)$ & $(25.63)$ & $(6.72)$ & $(22.67)$ \\
$\mathrm{f}_{3}$ & $3.08 \mathrm{e}-14$ & 6.057 & $\mathbf{7 . 9 9 e - 1 5}$ & $5.84 \mathrm{e}-13$ & $\mathbf{7 . 9 9 e - 1 5}$ \\
& $(1.72 \mathrm{e}-14)$ & $(9.49)$ & $(0)$ & $(2.10 \mathrm{e}-13)$ & $(0)$ \\
$\mathrm{f}_{4}$ & $\mathbf{9 . 5 3 e - 1 6}$ & $2.17 \mathrm{e}-2$ & $3.69 \mathrm{e}-4$ & $\mathbf{8 . 9 6 e - 1 6}$ & $1.84 \mathrm{e}-4$ \\
& $(7.70 \mathrm{e}-17)$ & $(2.05 \mathrm{e}-2)$ & $(1.65 \mathrm{e}-3)$ & $(1.18 \mathrm{e}-16)$ & $(1.16 \mathrm{e}-3)$ \\
$\mathrm{f}_{5}$ & $\mathbf{9 . 3 6 e - 3 1}$ & $2.07 \mathrm{e}-2$ & $1.31 \mathrm{e}-30$ & $8.69 \mathrm{e}-24$ & $\mathbf{9 . 2 0 e}-31$ \\
& $(6.04 \mathrm{e}-32)$ & $(7.21 \mathrm{e}-2)$ & $(7.83 \mathrm{e}-31)$ & $(8.46 \mathrm{e}-24)$ & $(6.98 \mathrm{e}-32)$ \\
$\mathrm{f}_{6}$ & $\mathbf{7 . 1 6 e - 3 1}$ & $1.64 \mathrm{e}-3$ & $4.41 \mathrm{e}-30$ & $3.15 \mathrm{e}-23$ & $\mathbf{8 . 9 7 e - 3 1}$ \\
& $(7.75 \mathrm{e}-32)$ & $(4.02 \mathrm{e}-3)$ & $(4.03 \mathrm{e}-30)$ & $(2.44 \mathrm{e}-23)$ & $(8.32 \mathrm{e}-32)$ \\
$\mathrm{f}_{7}$ & $\mathbf{- 9 7 . 4 6}$ & -63.67 & -30.21 & -35.92 & -39.34 \\
& $(4.04 \mathrm{e}-2)$ & $(4.45)$ & $(1.97)$ & $(0.73)$ & $(1.76)$ \\
$\mathrm{f}_{11}$ & $\mathbf{- 7 8 . 3 3}$ & -59.24 & -77.14 & -78.29 & -69.13 \\
& $(3.97 \mathrm{e}-10)$ & $(1.54)$ & $(6.39 \mathrm{e}-1)$ & $(7.67 \mathrm{e}-2)$ & $(5.75)$ \\
$\mathrm{f}_{8}$ & $1.56 \mathrm{e}-1$ & $4.68 \mathrm{e}+3$ & $\mathbf{4 . 0 0 e}-2$ & 4.26 & 5.14 \\
& $(2.09 \mathrm{e}-1)$ & $(4.47 \mathrm{e}+3)$ & $(6.65 \mathrm{e}-2)$ & $(6.84)$ & $(6.02)$ \\
$\mathrm{f}_{9}$ & $5.54 \mathrm{e}-27$ & $4.78 \mathrm{e}-48$ & $4.86 \mathrm{e}-30$ & $4.34 \mathrm{e}-24$ & $\mathbf{8 . 2 1 e - 5 8}$ \\
& $(1.38 \mathrm{e}-26)$ & $(1.44 \mathrm{e}-47)$ & $(4.53 \mathrm{e}-30)$ & $(2.28 \mathrm{e}-24)$ & $(1.70 \mathrm{e}-57)$ \\
$\mathrm{f}_{10}$ & $2.02 \mathrm{e}-14$ & $\mathbf{8 . 8 8 e - 3 2}$ & $7.11 \mathrm{e}-15$ & $3.36 \mathrm{e}-14$ & $4.83 \mathrm{e}-18$ \\
& $(5.77 \mathrm{e}-14)$ & $(1.94 \mathrm{e}-31)$ & $(4.64 \mathrm{e}-15)$ & $(8.63 \mathrm{e}-15)$ & $(6.20 \mathrm{e}-18)$ \\
\hline & & & & &
\end{tabular}

From the obtained results, it is clear that no algorithm performs superiorly better than others. But CLA-DE can achieve a near optimal solution in all cases. From this perspective, it is superior to all the other compared algorithms because any one of them fails to achieve a reasonable result on some of the benchmark functions. For $\mathrm{f}_{1}, \mathrm{f}_{2}, \mathrm{f}_{7}$ and $\mathrm{f}_{8}$ CLA-DE obtained the least mean best fitness values. Considering figures 7(a) and 8, it also achieved these results with the highest convergence rate. For $\mathrm{f}_{3}, \mathrm{f}_{5}$, and $\mathrm{f}_{6}$ it also achieved the near best results, but with a lower rate than ODE. However, its convergence rate is acceptable and isn't far worse than that of ODE for these functions. It also has good convergence rate for these functions in comparison to algorithms other than ODE (figure 7).

CLA-DE presents some compromise between local search and global search. Because it considers the whole search space, and each parameter can take values from the entire space, it is immune to entrapment in local optima. It is primarily based on meta-intelligent mutations, which diversify its population, and so it does not have the problem of stagnation and premature convergence even in the case of 
small populations $(3 \times 4$ CLA in our experiments). Due to this characteristic, it shows a slower convergence rate on functions $\mathrm{f}_{10}$ and $\mathrm{f}_{11}$.

\section{Conclusion}

In this paper, a hybrid method based on cellular learning automata and deferential evolution algorithm is presented for solving global optimization problems. The methodology relies on evolving some models of the search space. Each Solution Model is composed of a group of learning automata and is placed in one cell of a $\mathrm{CA}$, and so search space is dynamically divided into some stochastic areas corresponding to the actions of learning automata. These learning automata interacted with each other through some CA rules. Also, for better commutation and finer results, DE algorithm was incorporated.

CLA-DE was compared to some other global optimization approaches tested on some benchmark functions. Results exhibited the effectiveness of the proposed model in achieving finer results. It was able to find the global optima on almost all of the test functions with acceptable accuracy. In some cases, it showed slower convergence rate than the best algorithms, but the difference between its results and the best ones is admissible. The proposed method totally exhibits good global search capability with an acceptable convergence rate.

As future works, working on fuzzy actions for learning automata to improve searching at boundary points of actions will be interesting. Also, considering other learning algorithms for the learning automata might be beneficial.

\section{References}

[1] Eiben AE, Smith JE (2003) Introduction to evolutionary computing. Springer, New York

[2] Howell MN, Gordon TJ, Brandao FV (2002) Genetic learning automata for function optimization. IEEE Trans Syst Man Cybern 32:804-815

[3] Zeng X, Liu Z (2005) A learning automata based algorithm for optimization of continuous complex functions. Inf Sci 174:165-175

[4] Hong J, Prabhu VV (2004) Distributed reinforcement learning control for batch sequencing and sizing in just-in-time manufacturing systems. J Appl Intell 20: 71-87

[5] Iglesias A, Martinez P, Aler R, Fernandez F (2009) Learning teaching strategies in an adaptive and intelligent educational system through reinforcement learning. J Appl Intell 31: 89-106

[6] Wiering MA, Hasselt HV (2008) Ensemble algorithms in reinforcement learning. IEEE Trans Syst Man Cybern, Part B 38(4): 930-936 
[7] Narendra KS, Thathachar MAL (1989) Learning automata: an introduction. Prentice Hall, New Jersey

[8] Tathachar M AL, Sastry PS (2002) Varieties of learning automata: an Overview”, IEEE Transaction on Systems, Man, and Cybernetics-Part B: Cybernetics, Vol. 32, No. 6, PP. 711-722, 2002.

[9] Najim K, Poznyak AS (1994) Learning automata: theory and applications. Pergamon, New York

[10] Thathachar MAL, Sastry PS (2003) Networks of learning automata: techniques for online stochastic optimization. Springer-Verlag, New York

[11] Haleem MA, Chandramouli R (2005) Adaptive downlink scheduling and rate selection: a cross-layer design. IEEE J Sel Areas Commun 23:1287-1297

[12] Sastry PS, Nagendra GD, Manwani N (2010) A team of continuous-action learning automata for noise-tolerant learning of half-spaces. IEEE Trans Syst Man Cybern, Part B: Cybern 40(1): 1928

[13] Granmo OL, Oommen BJ (2010) Optimal sampling for estimation with constrained resources using a learning automaton-based solution for the nonlinear fractional knapsack problem. J Appl Intell 33: 3-20

[14] Meybodi MR, Beigy H, Taherkhani M (2003) Cellular learning automata and its applications. Sharif J Sci technol 19:54-77

[15] Esnaashari M, Meybodi MR (2008) A cellular learning automata based clustering algorithm for wireless sensor networks. Sens Lett 6(5):723-735

[16] Esnaashari M, Meybodi MR (2010) Dynamic point coverage problem in wireless sensor networks: a cellular learning automata approach. J Ad Hoc \& Sens Wirel Netw 10(2-3):193-234 [17] Talabeigi M, Forsati R, Meybodi MR (2010) A hybrid web recommender system based on cellular learning automata. In Proc 2010 IEEE Int Conf Granul Comput, San Jose, California, 453458

[18] Beigy H, Meybodi MR (2008) Asynchronous cellular learning automata. J Automatica 44(5):1350-1357

[19] Beigy H, Meybodi MR (2010) Cellular learning automata with multiple learning automata in each cell and its applications. IEEE Trans Syst Man Cybern, Part B: Cybern 40:54-66

[20] Beigy H, Meybodi MR (2006) A new continuous action-set learning automata for function optimization. J Franklin Inst 343:27-47

[21] Rastegar R, Meybodi MR, Hariri A (2006) A new fine-grained evolutionary algorithm based on cellular learning automata. Int J Hybrid Intell Syst 3:83-98

[22] Hashemi AB, Meybodi MR (2011) A note on the learning automata based algorithms for adaptive parameter selection in PSO. Appl Soft Comput 11:689-705

[23] Storn R, Price K (1995) Differential evolution - a simple and efficient adaptive scheme for global optimization over continuous spaces. Technical report, International Computer Science Institute, Berkley

[24] Storn R, Price KV (1997) Differential evolution- a simple and efficient heuristic for global optimization over continuous Spaces. J Glob Optim 11:341-359 
[25] Kaelo P, Ali MM (2006) A numerical study of some modified differential evolution algorithms. Eur J Oper Res 169:1176-1184

[26] Brest J, Greiner S, Boskovic B, Mernik M, Zumer V (2006) Self adapting control parameters in differential evolution: a comparative study on numerical benchmark problems. IEEE Trans Evol Comput 10(6):646-657

[27] Rahnamayan S, Tizhoosh HR, Salama MMA (2008) Opposition-based differential evolution. IEEE Trans Evol Comput 12:64-79

[28] Brest J, Maucec MS (2008) Population size reduction for the differential evolution algorithm. J Appl Intell 29: 228-247

[29] Chakraborty UK (2008) Advances in differential evolution. Springer-Verlag, Heidelberg Germany, 2008

[30] Vesterstrom J, Thomsen R (2004) A comparative study of differential evolution, particle swarm optimisation and evolutionary algorithms on numerical benchmark problems. In: Proc Sixth Congr Evol Comput (CEC-2004), IEE Press, Piscataway, NJ, USA, 1980-1987

[31] Wolfram S (1994) Cellular automata and complexity. Perseus Books Group

[32] Leung YW, Wang YP (2001) An orthogonal genetic algorithm with quantization for global numerical optimization. IEEE Trans Evol Comput 5:41-53

[33] Yao X, Liu Y, Lin G (1999) Evolutionary programming made faster. IEEE Trans Evol Comput 3:82-102

[34] Shi Y, Eberhart RC (1999) Empirical study of particle swarm optimization. In Proc IEEE Int Congr Evol Comput, (CEC 1999), Piscataway, NJ, IEEE Press 3:101-106

[35] Srorn R, Differential evolution (DE) for continuous function optimization. Rainer's Homepage. http://www.icsi.berkeley.edu/ storn/code.html. Accessed January 2010

[36] Liu J, Lampinen J (2005) A fuzzy adaptive differential evolution algorithm. Soft Comput - A Fusion Found Methodol Appl 9: 448-462 


\section{Figure legends}

Figure 1 A scheme of CLA-DE model entities

Figure 2 General pseudo code for CLA-DE

Figure 3 (a) Learning automata of each cell is randomly partioned into exclusive groups and each group creates one child for the cell. (b) Reinforcement signals from generated children and the neighboring cells are used for updating the Solution Model and the best generated child is used for updating the Candidate Solution.

Figure 4 pseudo code for Model Based Evolution phase

Figure 5 pseudo code for action refinement step

Figure 6 pseudo code for DE Based Evolution

Figure 7 Fitness curves for functions (a) f2, (b) f3, (c) f4, (d) f5, (e) f6, (f) f9, (g) f10 and (h) f1 1. The horizontal axis is the number of fitness evaluations and the vertical axis is the logarithm in base 10 of mean best function value over all trials.

Figure 8 Fitness curves for functions (a) $\mathrm{fl}$, (b) $\mathrm{f} 7$ and (c) $\mathrm{f} 8$. The horizontal axis is the number of fitness evaluations and the vertical axis is the mean best function value over all trials.

\section{table title}

Table 1 benchmark functions and their properties

Table 2 Mean success rate and Mean number of function evaluations to reach the specified accuracy for successful runs. Success rates are shown inside the parentheses

Table 3 Success rate and Mean number of function evaluation ranks of the compared methods. Success rate ranks are shown inside the parentheses

Table 4 average final result (along with its standard deviation) 